\title{
NATURAL SURFACE LAYER OF METALS
}

\author{
G. Starzyński and W. Gambin \\ Institute of Fundamental Technological Research, Polish Academy of Sciences \\ Świętokrzyska 21, 00-049 Warsaw, Poland
}

A notion of natural surface layer of metals as a reference state for a mechanical description of technological surface layers is introduced. According to experimental observations it is assumed that the yield stress of metal surface layer without any technological treatment is lower than in the bulk material. A simple mechanical model that enables one to find a yield stress distribution in the natural surface layer is proposed. As an example the natural surface layer of aluminium specimen is examined. According to the model and experimental observations, a layer thickness is equal 2-3 grains' diameters, and the yield stress in the layer may be $30 \%$ lower than in the bulk material.

PACS numbers: $68.10 . \mathrm{Cr}, 68.35 . \mathrm{Gy}$

\section{Introduction}

In the years' $50-60$, metal surface layers without any technological treatment were extensively examined [1-2]. Measurements based on X-ray research revealed a difference between the yield stress in the surface layer and that in the bulk material. The hypothesis about early yielding of subsurface grains was proposed and next confirmed experimentally [3-5]. A strong work hardening of the layers as a result of their early yielding was observed and described in the literature [6-8].

A strength of machine elements may be considerably improved by a formation of technological surface layers. To distinguish them from those without any technological treatment, the last ones are called the natural surface layers. It is necessary to distinguish the natural surface layers from the physical surface layers of monocrystals [9]. The aim of our work is to introduce and to examine the notion of the natural surface layer as a reference state for a mechanical description of technological surface layers.

\section{Yield stresses of mono- and polycrystal}

Let us consider a tensioned monocrystalline specimen and assume that the critical shear stress $\tau_{\mathrm{c}}$ on the active slip system is known. The average yield stress $\bar{\sigma}_{c}$ for all the possible orientations of the monocrystal is connected with $\tau_{c}$ by the rule: $\bar{\sigma}_{\mathrm{c}}=m \tau_{\mathrm{c}}$. If $\tau_{\mathrm{c}}$ is the same for all slip systems of the considered monocrystal, the parameter $m$ is equal to 2.238 (see Ref. [10]). 


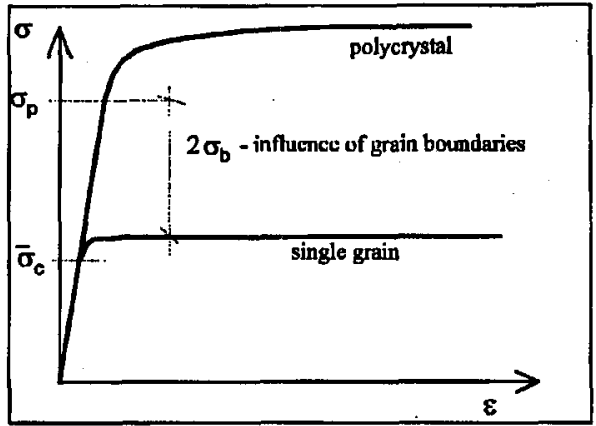

Fig. 1. Difference between yield stresses of mono- and polycrystal.

Now, let us consider a tension of an isotropic polycrystalline specimen composed of the grains with the average yield stress $\bar{\sigma}_{\mathrm{c}}$. The measured yield stress $\sigma_{\mathrm{p}}$ is considerably higher than $\bar{\sigma}_{\mathrm{c}}$ (Fig. 1 ). The difference denoted by $2 \sigma_{\mathrm{b}}$ determines an influence of grain boundaries on the yield stress of polycrystal.

Generally: $\sigma_{\mathrm{p}}=M \tau_{\mathrm{c}}$, where $M>m$. For fcc metals Taylor has obtained $M$ equal to 3.06 (see Ref. [11]). The result of his calculation is in good agreement with experiments for a pure aluminium. In this case the ratio $\sigma_{\mathrm{p}} / \bar{\sigma}_{\mathrm{c}}$ is equal to 1.5. This ratio may be higher for other metals.

\section{Mechanism of plastic yield for polycrystalline grains}

Let us consider a metal element composed of randomly distributed grains of the same diameter: Let us cut a specimen from the above element so that any residual stresses, cracks and impurities are not introduced. The surface layer of the specimen will be called its natural surface layer. Let us decompose this layer onto sublayers of one grain's thickness, as it is shown in Fig. 2.

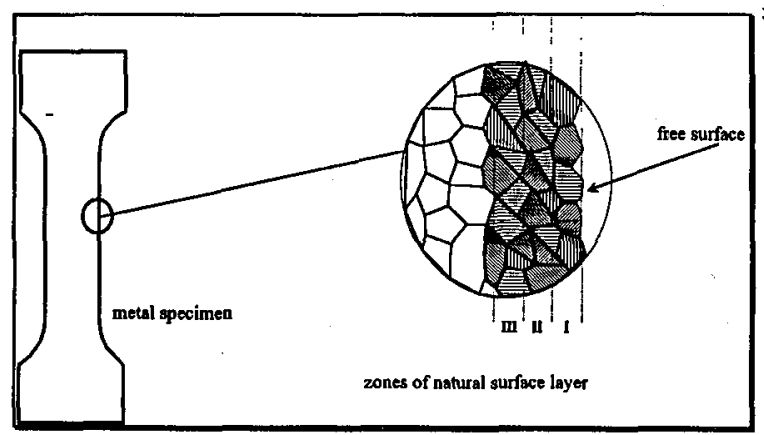

Fig. 2. Natural surface layer of polycrystalline specimen. 
Because of weaker energy barriers for dislocations on a specimen free surface, the number of moving dislocation in the surface sublayer is higher than in the remaining sublayers. A plastic yield in this sublayer weakens dislocation barriers on the boundary with the next sublayer. As a consequence all the successive sublayers yield earlier than the material inside the specimen. A thickness of the natural surface layer is determined by the number of sublayers with the yield stress lower than $\sigma_{\mathrm{p}}$.

\section{Yield stress distribution within natural surface layer}

Far from the surface of the specimen, the yield stress is assumed to be

$\sigma_{\mathrm{p}}=\bar{\sigma}_{\mathrm{c}}+2 \sigma_{\mathrm{b}}$

where $\bar{\sigma}_{\mathrm{c}}$ is the average yield stress for a single grain and $2 \sigma_{\mathrm{b}}$ is an additional stress needed to overcome grain boundaries.

Let us consider the sublayers of the natural surface layer. They form zones that are numbered successively from the free surface to the inside of the specimen. To determine their yield stress it is necessary to find the additional stresses $2 \sigma_{\mathrm{b}}$, which are different in different zones. For a given zone, divide the stress $2 \sigma_{b}$ onto two parts: the one caused by the boundary with the inner neighbouring zone, and the other caused by the outer one. Assume that the first part of the stress $2 \sigma_{\mathrm{b}}$ for all zones is the same and equal to $\sigma_{\mathrm{b}}$. The second part denoted by $\sigma_{\text {ext }}^{(K)}$ (where $K=1,2, \ldots, N)$ is smaller and it increases in the successive zones from zero to a value close to $\sigma_{\mathrm{b}}$ in the last zone of the natural surface layer. Then, the yield stresses $\sigma^{(K)}$ in the successive zones are the following (Fig. 3):

$$
\left\{\begin{array}{l}
\sigma^{(1)}=\bar{\sigma}_{\mathrm{c}}+\sigma_{\mathrm{b}} \\
\sigma^{(K)}=\bar{\sigma}_{\mathrm{c}}+\sigma_{\mathrm{b}}+\sigma_{\mathrm{ext}}^{(K)} \quad \text { for } \quad K=2,3, \ldots, N .
\end{array}\right.
$$

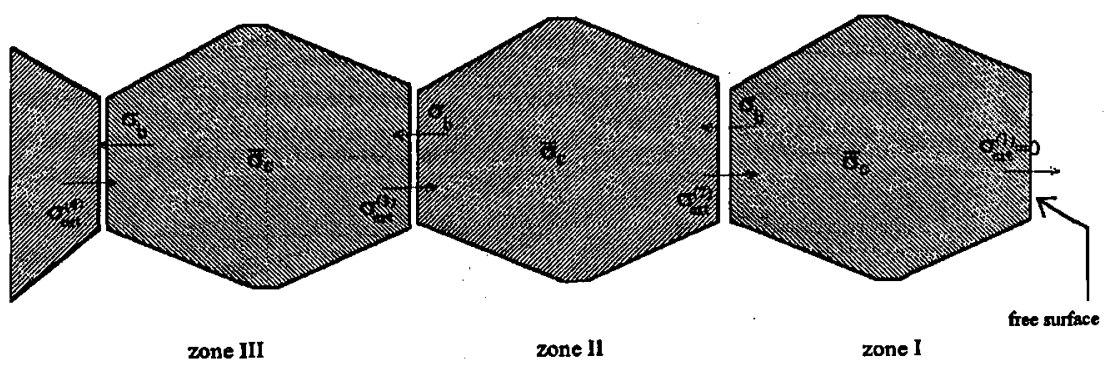

Fig. 3. Yield stress decomposition in zones of natural surface layer.

To determine $\sigma_{\text {ext }}^{(K)}$ the following hypothesis is postulated:

HYPOTHESIS: The additional stress $\sigma_{\mathrm{ext}}^{(K)}$ depends on the yield stress of the $(K-1)$-th grain and the yield stress of polycrystal, according to the rule: $\sigma_{\text {ext }}^{(K)}=$ $\sigma^{(K-1)} \sigma_{\mathrm{b}} / \sigma_{\mathrm{p}}$, with $\sigma_{\mathrm{ext}}^{(1)}=0$. 
Introducing the above rule and the relation (1) into (2), one can obtain the following recurrent expression:

$$
\sigma^{(K)}=\bar{\sigma}_{\mathrm{c}}+\frac{1}{2}\left(\sigma_{\mathrm{p}}-\bar{\sigma}_{\mathrm{c}}\right)\left(1+\frac{\sigma^{(K-1)}}{\sigma_{\mathrm{p}}}\right)
$$

for $K=1,2, \ldots, N$ and $\sigma^{(0)}=0$. The above relation describes the yield stress distribution within the natural surface layer, if the yield stress $\sigma_{\mathrm{p}}$ for a polycrystalline specimen and the average yield stress $\bar{\sigma}_{c}$ for a single grain are known.

Let us consider the natural surface layer for a pure aluminium. As it was mentioned in Sec. 1 , in this case the ratio $\sigma_{\mathrm{p}} / \bar{\sigma}_{\mathrm{c}}$ is equal to 1.5 . One can calculate from the relation (3) that the ratios $\sigma^{(K)} / \sigma_{\mathrm{p}}$ for $K=1,2,3,4$ are equal successively: $0.866,0.982,0.998,1.00$. Note that the difference between $\sigma^{(K)}$ and $\sigma_{\mathrm{p}}$ reaches $13.4 \%$ in the first zone, it is smaller than $2.0 \%$ in the second zone and it decreases $0.2 \%$ in the third zone. Assuming the accuracy of the calculation $0.1 \%$, one can determine the thickness of natural surface layer being equal three grain diameters.

The value $\sigma^{(K)}$ is considered as a value of the yield stress at the middle of $K$-th zone. Let us denote by $\sigma^{\langle K\rangle}$ and $\sigma^{\langle K-1\rangle}$ the yield stress at the outer and the inner boundary of the $K$-th zone, respectively. Assuming the linear yield stress distribution within each zone, the following relation is satisfied: $\sigma^{(K)}=$ $\frac{1}{2}\left(\sigma^{(K)}+\dot{\sigma}^{\langle K-1\rangle}\right)$. In the considered case, the ratios $\sigma^{(K)} / \sigma_{\mathrm{p}}$ for $K=0,1,2,3$ are equal successively to: $0.764,0.968,0.996,1.00$. The $24 \%$ decrease in the yield stress is observed on the specimen surface. The results are shown in Fig. 4.

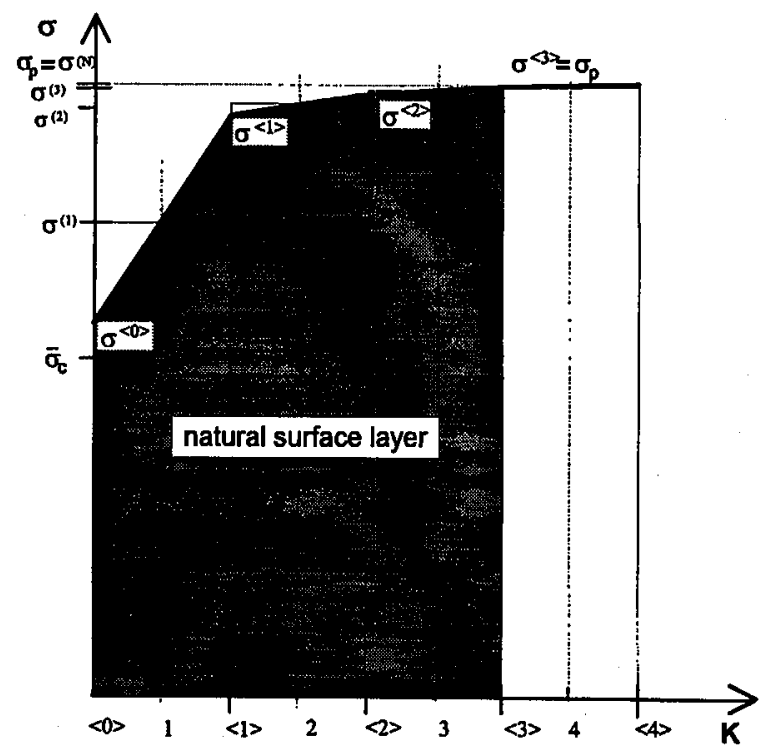

Fig. 4. Yield stress distribution in natural surface layer. 
The X-ray measurements of carbon steel indicate that the $30 \%$ decrease in the yield stress occurs in the $0.01 \mathrm{~mm}$ thick surface layer [1].

\section{Conclusions}

According to many experimental investigations, the yield stress in surface layers before their technological treatment is lower than in the bulk material. For mechanical description of technological surface layers, the notion of the natural surface layer is introduced. It is assumed that weak dislocation barriers on free surfaces of metal elements are responsible for the low yield stress in the natural surface layer. The yield stress in the layer is expressed as a function of the yield stress for polycrystalline specimen, the yield stress for a single grain and a distance from the free surface of metal element. Detailed calculations are performed for a pure aluminium specimen. For this case, the thickness of natural surface layer is equal to three grain diameters and the yield stress decreases $24 \%$ on the free surface of the layer.

\section{Acknowledgment}

The paper was supported by the Committee for Scientific Research (grant PB3P40201607).

\section{References}

[1] L.A. Glikman, T.P. Sariflowa, W.A. Stiepanov, Zh. Tekh. Fiz. 19, 3 (1949) (in Russian).

[2] K. Sumino, H. Yamamoto, J. Phys. Soc. Jpn. 16, 1 (1961).

[3] E.L. Assur, N.N. Davidenkov, Zh. Tekh. Fiz. 19, 10 (1949) (in Russian).

[4] R. Prummer, E. Macherauch, Z. Naturforsch. A 20, 7 (1965).

[5] B. Greenough, J. Iron \& Steel 196, 3 (1951).

[6] W.J. Szabalin, Dokl. Akad. Nauk SSSR 144, 3 (1962) (in Russian).

[7] W.F. Terentiev, N.S. Ivanova, Fiz. Khim. Obrab. Mater. 1, (1970) (in Russian).

[8] W.F. Terentiev, Dokl. Akad. Nauk SSSR 185, 1 (1969) (in Russian).

[9] W.P. Alehin, Physics of Strength and Plasticity of Surface Layers of Metals, Nauka, Moscow 1983 (in Russian).

[10] G. Sachs, Z. D. Ver. Deut. Ing. 72, 734 (1928).

[11] G.I. Taylor, Proc. R. Soc. A 145, 362 (1934). 